\title{
Microbiological Profile of Blood Stream Infections in Febrile Neutropenic Patients at a Tertiary Care Teaching Hospital in Rishikesh, Uttarakhand
}

\author{
Manisha Paul ${ }^{1} \quad$ Mohit Bhatia ${ }^{1, \odot}$ Udayakumar Sasi Rekha ${ }^{1} \quad$ Diksha $^{1} \quad$ Balram Ji Omar ${ }^{1}$ Pratima Gupta \\ ${ }^{1}$ Department of Microbiology, All India Institute of Medical \\ Sciences, Rishikesh, Uttarakhand, India

\begin{abstract}
Address for correspondence Mohit Bhatia, MBBS, MD, DTM\&H, Department of Microbiology, All India Institute of Medical Sciences, Rishikesh 249203, Uttarakhand, India (e-mail: docmb1984@gmail.com).
\end{abstract}

J Lab Physicians:2020;12:147-153

\begin{abstract}
Keywords

- febrile neutropenia

- BSIs

- hematological malignancies

- gram-negative bacilli
\end{abstract}

Context Febrile neutropenia is a serious complication of chemotherapy affecting patients with both hematological and solid malignancies, respectively. To the best of our knowledge, there is paucity of literature from Uttarakhand, India on microbiological profile of blood stream infections (BSIs) in febrile neutropenic patients.

Aims The study aims to generate preliminary data on microbiological profile and antibiotic resistance pattern of BSIs in febrile neutropenic patients.

Settings and Design The design involved cross-sectional study from January 1, 2019 to July 31, 2019.

Methods and Materials Data of nonrepetitive paired peripheral blood samples obtained from 306 consecutive febrile neutropenic cancer patients of all age groups and both sexes, for culture and sensitivity testing, were retrospectively analyzed. All blood samples were subjected to aerobic culture using BACT/ALERT three-dimensional microbial detection system. Growth obtained in culture was identified by conventional biochemical methods. Antibiotic susceptibility testing of bacterial isolates was performed using modified Kirby Bauer disk diffusion method.

Statistical Analysis Used Fisher's exact test was used for the analysis.

Results Mean age \pm SD of the study population was $32.39 \pm 10.56$ years with a male to female ratio of 1.55:1. 74.18\% of the blood samples were received from patients suffering from hematological malignancies. Microbiologically confirmed BSIs were observed in $27.1 \%$ patients. Gram-negative bacilli were predominantly isolated in culture with Klebsiella spp. being the most common. Percentage resistance values of gram-negative bacilli to aminoglycosides, $\beta$-lactam/ $\beta$-lactamase inhibitor combinations, fluoroquinolones, cephalosporins, carbapenems, chloramphenicol, ampicillin, co-trimoxazole, and doxycycline were 26.6 to $91.7 \%, 8.3$ to $86.6 \%, 10$ to $66.7 \%, 13.3$ to $73.3 \%, 8.3$ to $73.3 \%$, 80 to $93.3 \%, 13.3$ to $20 \%, 16.7$ to $66.6 \%$, and 13.3 to $16.7 \%$, respectively.

Conclusion Implementation of antimicrobial stewardship program along with hospital infection control practices is needed for preventing BSIs due to MDR organisms.

\section{Introduction}

Febrile neutropenia (FN) or neutropenic fever is defined as a single oral temperature of $\geq 38.3^{\circ} \mathrm{C}\left(101^{\circ} \mathrm{F}\right)$ or temperature of $\geq 38.0^{\circ} \mathrm{C}\left(100.4^{\circ} \mathrm{F}\right)$ sustained over a 1-hour period along with an absolute neutrophil count (ANC) of $<500$ cells $/ \mathrm{mm}^{3}$, or an
ANC that is expected to decrease to $<500$ cells $/ \mathrm{mm}^{3}$ during the next 48 hours. ${ }^{1}$ It is a serious complication of chemotherapy affecting $80 \%$ and 10 to $50 \%$ of those with hematological and solid malignancies, respectively. ${ }^{1,2}$ Despite major advances in medical management, FN is associated with prolonged hospital stays, increased health care costs, compromised efficacy
DOI https://doi.org/ 10.1055/s-0040-1716661 ISSN 0974-2727.
(C)2020 by The Indian Association of Laboratory Physicians
License terms

(우 (1) $\Theta \circledast$ 
of chemotherapy, increased morbidity, and mortality rates, respectively. ${ }^{3}$

The possibility of blood stream infections (BSIs) is a major cause of concern in these patients. BSIs are responsible for 10 to $25 \%$ of all febrile episodes in neutropenic patients, with an incidence as high as 13 to $60 \%$ in hematopoietic stem cell transplantation recipients. Severe sepsis and septic shock have been, respectively estimated to occur in 20 to $30 \%$ and 5 to $10 \%$ of FN patients. ${ }^{1,4-7}$ Crude mortality rates reaching $40 \%$ have been reported in FN patients with underlying hematological malignancies. ${ }^{8}$ Although, there has been a substantial reduction in mortality rates in neutropenic patients with BSIs from $25 \%$ in late 1970 s to $6 \%$ in the recent years, increased prevalence of multidrug-resistant (MDR) bacterial infections may hamper this progress, as has been highlighted by several authors. ${ }^{9-12}$

There has been a paradigm shift in the spectrum of microorganisms isolated from FN patients over the last 40 years. Until mid-1980s, Escherichia coli, Klebsiella spp., and Pseudomonas aeruginosa among gram-negative bacilli and Staphylococcus aureus among gram-positive cocci, respectively, were the most frequently isolated organisms from this group of patients. These organisms have gradually been replaced by coagulase-negative staphylococci (CoNS) and viridans group of streptococci ever since. ${ }^{3}$ In the late 1970 s and early 1980s, gram-negative bacilli were the predominant cause of BSIs in neutropenic patients which were gradually replaced by gram-positive cocci in the $1990 \mathrm{~s} .^{13}$ Several studies published in the recent years have reported a shift in prevalence from gram-positive to gram-negative bacteremia with an accompanying rise and successful dissemination of MDR gram-negative bacterial infections. ${ }^{8,14}$

Keeping in mind the ever-changing microbiological profile of BSIs in FN patients, it is imperative that data about the locally prevalent pathogens and their antimicrobial resistance patterns should be generated and analyzed to decide appropriate empirical therapy. The present study was conducted with the aim of generating preliminary data on microbiological profile and antibiotic resistance pattern of BSIs in FN cancer patients. To the best of our knowledge, this study is the first of its kind from the state of Uttarakhand, India. The findings of this study will be of immense help to treating physicians in this part of the country, in instituting timely and appropriate antimicrobial therapy in this group of patients.

\section{Subjects and Methods}

A cross-sectional study was conducted at a tertiary care teaching hospital located in Rishikesh, Uttarakhand, for a time period of 7 months from January 1, 2019 to July 31, 2019. Data of nonrepetitive paired peripheral blood samples obtained from 306 consecutive FN cancer patients of all agegroups and both sexes, for culture and sensitivity testing, were retrospectively analyzed. This study was approved by Institutional Ethics Committee vide letter number AIIMS/ IEC/19/1275 dated December 31, 2019. A total of 306 episodes of FN (one per patient) were studied. Each incident of FN was treated as a separate episode. An episode of bacteremia was defined as greater than or equal to one blood culture yielding a pathogen, with the exception of CoNS, for which isolation from two blood culture samples was considered significant.

All blood samples had been subjected to aerobic culture using BACT/ALERT 3D microbial detection system (bioMerieux India Private Limited). The blood culture bottles which beeped positive were subjected to preliminary microscopic examination of gram-stained smears of their contents and the concerned treating physicians were informed telephonically. Subcultures were also performed from these bottles on blood and MacConkey agar plates, respectively, which were incubated aerobically at $37^{\circ} \mathrm{C}$ overnight. Growth obtained on culture plates was studied on the following day and subjected to further identification by conventional (manual) biochemical methods. ${ }^{15}$ Antibiotic susceptibility testing of bacterial isolates was performed using modified Kirby Bauer disk diffusion method, the results of which were interpreted as per Clinical and Laboratory Standards Institute guidelines 2019. ${ }^{15,16}$ American Type Culture Collection control strains were used to ensure the quality of each procedure.

Categorical variables were presented as proportions while continuous variables were presented as mean with standard deviation. Comparison of categorical variables was done by Fisher's exact test. All statistical tools were two-tailed and a significant level $p<0.05$ was used. All statistical tests were performed using InStat software (GraphPad Software, San Diego, California, United States).

\section{Results}

(1) Baseline characteristics: During the study period, $60.8 \%$ (186/306) and 39.2\% (120/306) of the paired peripheral blood samples were from male and female patients, respectively, with a male to female ratio of 1.55:1. Mean age \pm S.D. of these patients was $32.39 \pm 10.56$ years, with maximum number of cases occurring in 0 to 10 [62/306 (20.2\%)] followed by 21 to 30 [61/306 (19.9\%)] years of age groups, respectively. One hundred and fifty-four (50.3\%), 121 (39.5\%), and 31 (10.1\%) of these samples were received from Haemato-Oncology, Medical Oncology, and Surgical Oncology wards, respectively.

(2) Clinical diagnosis: Most of the blood samples were received from patients suffering from hematological malignancies (227/306 [74.18\%]), with acute leukemia being the most common diagnosis (185/306 [60.46\%]). Overall frequency distribution of FN patients with respect to clinical diagnosis has been depicted in $\boldsymbol{- F i g}$. 1 . - Tables $\mathbf{1}$ and $\mathbf{2}$, respectively summarize the frequency distribution of FN among different types of hematological and nonhematological malignancies.

(3) Microbiological profile: While $66.6 \%(204 / 306)$ of the paired blood samples were sterile, laboratory contaminants were grown in culture from 18.6\% (19/102) of the remaining samples. Growth of microorganisms with potential clinical significance, was obtained in culture from $81.3 \%$ (83/102) of the samples, which included 54 gram-negative bacilli, 21 gram-positive cocci, and eight fungal isolates, respectively, as enumerated in - Table 3. 


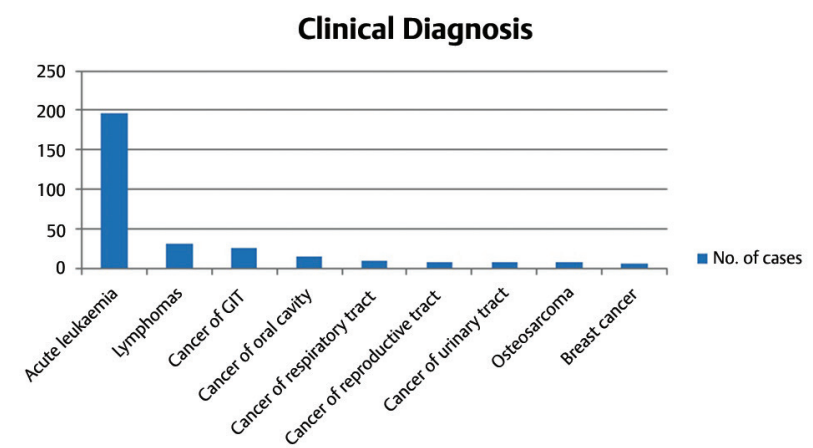

Fig. 1 Overall frequency distribution of febrile neutropenic patients with respect to clinical diagnosis.

Table 1 Frequency distribution of febrile neutropenia among patients with different types of hematological malignancies $(N=227)$

\begin{tabular}{|l|l|}
\hline $\begin{array}{l}\text { Type of hematological } \\
\text { malignancy }\end{array}$ & $\begin{array}{l}\text { Febrile neutropenic } \\
\text { patients } \\
\text { n(\%) }\end{array}$ \\
\hline ALL & $79(34.80)$ \\
\hline B-cell ALL & $62(27.31)$ \\
\hline AML & $32(14.09)$ \\
\hline T-cell ALL & $12(5.28)$ \\
\hline NHL & $10(4.4)$ \\
\hline DLBCL & $9(3.96)$ \\
\hline CML & $9(3.96)$ \\
\hline HL & $4(1.76)$ \\
\hline Burkitt's lymphoma & $4(1.76)$ \\
\hline HGBCL & $3(1.32)$ \\
\hline T-cell LBL & $2(0.88)$ \\
\hline Splenic lymphoma & $1(0.44)$ \\
\hline
\end{tabular}

Abbreviations: ALL, acute lymphoblastic leukemia; AML, acute myeloid leukemia; CML, chronic myeloid leukemia; DLBCL, diffuse large B-cell lymphoma; HGBCL, LBL-lymphoblastic lymphoma; HL, Hodgkin lymphoma; NHL, non-Hodgkin lymphoma.

No statistically significant difference was observed between patients with hematological and nonhematological malignancies with respect to growth of potentially pathogenic microorganisms obtained in culture ( $p$-value:0.077; confidence interval [CI]: -0.004 to 0.21 ), type of microbial isolates obtained in culture (bacterial vs. fungal [ $p$-value: 0.34 ; $\mathrm{CI}$ : could not be calculated]; gram-negative bacilli vs. gram-positive cocci [ $p$-value: 0.33; CI: -0.082 to 0.32 ]), and gender ( $p$-value: 0.44 ; CI: 0.65-0.17), respectively.

(4) Antibiotic susceptibility: - Tables $\mathbf{4}$ and 5, respectively summarize the percentage antibiotic resistance pattern of predominant gram-negative bacilli (Klebsiella spp., E. coli, and Acinetobacter spp.) and gram-positive cocci (Coagulase negative Staphylococcus spp.).

Among the less frequently isolated bacterial isolates were Enterobacter spp. (3), P. aeruginosa (2), Citrobacter spp. (2), and Enterococcus spp. (1), respectively. Hundred
Table 2 Frequency distribution of febrile neutropenia among patients with different types of nonhematological malignancies $(N=79)$

\begin{tabular}{|l|l|}
\hline $\begin{array}{l}\text { Type of nonhematological } \\
\text { malignancy }\end{array}$ & $\begin{array}{l}\text { Febrile neutropenic } \\
\text { patients } \boldsymbol{n} \text { (\%) }\end{array}$ \\
\hline Ca gallbladder & $19(24.05)$ \\
\hline Ca oral cavity & $10(12.65)$ \\
\hline Ca urinary bladder & $7(8.86)$ \\
\hline Ca lung & $7(8.86)$ \\
\hline Ca breast & $5(6.33)$ \\
\hline Osteosarcoma & $4(5.06)$ \\
\hline Ca endometrium & $4(5.06)$ \\
\hline Ca ovary & $3(3.79)$ \\
\hline Ca stomach & $3(3.79)$ \\
\hline Ca pancreas & $2(2.53)$ \\
\hline Ca rectum & $2(2.53)$ \\
\hline Ca lower lip & $2(2.53)$ \\
\hline Ca larynx & $2(2.53)$ \\
\hline Ewing sarcoma & $2(2.53)$ \\
\hline Metastatic synovial sarcoma & $2(2.53)$ \\
\hline Ca tongue & $2(2.53)$ \\
\hline Ca buccal mucosa & $1(1.26)$ \\
\hline Nasopharyngeal carcinoma & $1(1.26)$ \\
\hline Abbrevation Cacance. & \\
\hline
\end{tabular}

Abbreviation: Ca, cancer.

percent in vitro resistance to ampicillin (Enterobacter spp. and Citrobacter spp.), fluoroquinolones (Citrobacter spp.), and co-trimoxazole (Citrobacter spp.), respectively was observed. Among the two P. aeruginosa isolates, one was pan sensitive and the other was resistant to aztreonam and gentamicin, respectively. Enterococcus species exhibited in vitro susceptibility to tigecycline, linezolid, chloramphenicol, and resistant to high level gentamicin $(120 \mu \mathrm{g})$, respectively. In vitro tigecycline susceptibility test results were interpreted as per European Committee on Antimicrobial Susceptibility Testing Guidelines (EUCAST) 2019. ${ }^{17}$

\section{Discussion}

Patients with underlying malignancies receiving cytotoxic antineoplastic therapy sufficient to adversely affect myelopoiesis and the developmental integrity of the gastrointestinal mucosa, are at risk for invasive infection due to microorganisms that translocate across intestinal mucosal surfaces. Fever may be the earliest and only sign of infection in neutropenic patients owing to muted neutrophil-mediated inflammatory response. Early recognition of neutropenic fever is critical for prompt initiation of empiric systemic antibacterial therapy to avoid progression to a sepsis syndrome and possibly death. ${ }^{18}$

Chemotherapy-induced mucositis occurs throughout the alimentary system. Seeding of the bloodstream with endogenous gastrointestinal tract flora is believed to cause the majority of episodes of FN. Also, administration of 
Table 3 Frequency distribution of microorganisms isolated from paired peripheral blood samples of febrile neutropenic patients $(N=83)$

\begin{tabular}{|l|l|l|l|}
\hline \multirow{2}{*}{ Organisms isolated } & \multicolumn{1}{|c|}{ Type of malignancy } \\
\cline { 2 - 4 } & $\begin{array}{l}\text { Hematological } \\
\text { malignancies } \\
n(\%)\end{array}$ & $\begin{array}{l}\text { Nonhematological } \\
\text { malignancies } \\
n(\%)\end{array}$ \\
\hline Klebsiella spp. & $18(21.7)$ & $2(2.4)$ & 20 \\
\hline Escherichia coli & $11(13.2)$ & $4(4.8)$ & 15 \\
\hline Acinetobacter spp. & $11(13.2)$ & $1(1.2)$ & 12 \\
\hline Citrobacter spp. & $2(2.4)$ & $0(0)$ & 2 \\
\hline Enterobacter spp. & $1(1.2)$ & $2(2.4)$ & 3 \\
\hline Pseudomonas aeruginosa & $2(2.4)$ & $0(0)$ & 2 \\
\hline Coagulase negative Staphylococcus spp. & $14(13.2)$ & $6(1.2)$ & 20 \\
\hline Enterococcus spp. & $1(1.2)$ & $0(0)$ & 1 \\
\hline Candida spp. & $7(10.3)$ & $0(0)$ & 7 \\
\hline Trichosporon spp. & $1(1.2)$ & $0(0)$ & 1 \\
\hline Total & $68(81.9)$ & $15(18.1)$ & 83 \\
\hline
\end{tabular}

chemotherapy not only decreases the number of neutrophils but also results in chemotactic and phagocytic defects. Immune defects related to underlying hematologic disorders, in addition to the immunosuppressive effects of chemotherapy, also place patients at higher risk for infection. ${ }^{18,19}$

In the present study, microbiologically confirmed BSIs were more commonly observed in male patients. Similar findings have been reported by various authors from different parts of the world. ${ }^{3,20-28}$ However, there is no specific explanation for this finding. The most common age group of patients in this study was 0 to 10 years which is in agreement with the study conducted by Kamonrattana et al..$^{28}$

More number of FN patients in our study, had underlying hematological as compared with nonhematological malignancies (solid tumors). These observations are in agreement with those of studies conducted by various authors. 3,20,23,24,28,29 It is a well-established fact that $\mathrm{FN}$ is more commonly observed in patients with hematological malignancies probably due to bone marrow dysfunction induced by underlying disease and chemotherapy.

Microbiologically confirmed BSIs were observed in 83 out of 306 patients (27.1\%). Similar observations were made by Mandal et al $^{30}$ The prevalence of microbiologically confirmed BSIs in FN patients with hematological and nonhematological malignancies was 22.2\% (68/306) and 4.9\% (15/306), respectively. The reported prevalence of BSIs in patients with hematological malignancies ranges from 11 to $38 \%{ }^{8}$ Low culture positivity rates in these patients may be explained in general by insufficient sample volume, prior administration of antibiotics, and provision of inappropriate culture conditions not suitable for growth of anaerobes and fastidious microorganisms, respectively. In the present study, all paired peripheral blood samples were subjected to aerobic culture only. Also, 22 out of 306 patients were on antibiotic therapy and blood cultures of 18 of these patients were sterile.

In this study, gram-negative bacilli were predominantly isolated in culture with Klebsiella spp. being the most common. Similar results were obtained in studies conducted by El-Mahallawy et al, Esbenshade et al, and Kokkayil et al. ${ }^{23-25,31}$ Among gram-positive bacteria, coagulase-negative staphylococci were the predominant isolates obtained in culture. Similar trend has also been reported by several other studies. $^{23-25,27,32}$

Varying resistance to different antibiotics was observed among gram-negative and positive bacteria, respectively. Among gram-negative bacilli, percentage resistance to aminoglycosides, $\beta$-lactam/ $\beta$-lactamase inhibitor combinations, fluoroquinolones, cephalosporins, carbapenems, chloramphenicol, ampicillin, co-trimoxazole, and doxycycline was 26.6 to $91.7 \%, 8.3$ to $86.6 \%, 10$ to $66.7 \%, 13.3$ to $73.3 \%, 8.3$ to $73.3 \%, 80$ to $93.3 \%, 13.3$ to $20 \%, 16.7$ to $66.6 \%$, and 13.3 to $16.7 \%$, respectively. In case of CoNS, percentage resistance to aminoglycosides, macrolides, fluoroquinolones, ampicillin, clindamycin, linezolid, doxycycline, chloramphenicol, and co-trimoxazole was 40\%, 35 to 50\%, 60 to $65 \%, 90 \%, 45 \%, 30 \%, 40 \%, 40 \%$, and 55\%, respectively. These findings are in concordance with the studies conducted by various authors. ${ }^{23,27,29,31,33-35}$ Blood stream infections due to antibiotic-resistant bacterial strains are on the rise in patients with neutropenic fever. The irrational use of broad-spectrum antibiotics as well as prolonged hospitalization may both add to the risk of acquiring infections with antibiotic-resistant bacteria. These pathogens are usually endogenous. Broad 
Table 4 Percentage antibiotic resistance pattern of predominant gram-negative bacterial isolates $(N 1=20, N 2=15, N 3=12)$

\begin{tabular}{|c|c|c|c|}
\hline Antibiotics & $\begin{array}{l}\text { Klebsiella spp. } \\
\text { n (\%) }\end{array}$ & $\begin{array}{l}\text { Escherichia coli } \\
n(\%)\end{array}$ & $\begin{array}{l}\text { Acinetobacter spp. } \\
n(\%)\end{array}$ \\
\hline \multicolumn{4}{|l|}{ Aminoglycosides } \\
\hline Amikacin & $6(30)$ & $4(26.6)$ & $7(58.3)$ \\
\hline Gentamicin & $8(40)$ & $8(53.3)$ & $11(91.7)$ \\
\hline \multicolumn{4}{|c|}{$\beta$-lactam/ $\beta$-lactamase inhibitors combinations } \\
\hline Amoxicillin-clavulanate & $7(35)$ & $13(86.6)$ & NA \\
\hline Piperacillin-tazobactam & $6(30)$ & $5(33.3)$ & $4(33.3)$ \\
\hline Ampicillin-sulbactam & $5(25)$ & $10(66.6)$ & $1(8.3)$ \\
\hline \multicolumn{4}{|l|}{ Fluoroquinolones } \\
\hline Ciprofloxacin & $2(10)$ & $2(13.3)$ & $8(66.7)$ \\
\hline Ofloxacin & $2(10)$ & $2(13.3)$ & NA \\
\hline \multicolumn{4}{|l|}{ Penicillins } \\
\hline Ampicillin & $16(80)$ & $14(93.3)$ & NA \\
\hline \multicolumn{4}{|l|}{ Cephalosporins } \\
\hline Cefuroxime & $11(55)$ & $8(53.3)$ & NA \\
\hline Cefotaxime & $13(65)$ & $11(73.3)$ & $5(41.6)$ \\
\hline Ceftriaxone & $10(50)$ & $10(66.6)$ & $2(16.6)$ \\
\hline Cefixime & $3(15)$ & $2(13.3)$ & NA \\
\hline Cefepime & $5(25)$ & $5(33.3)$ & $2(16.6)$ \\
\hline \multicolumn{4}{|l|}{ Carbapenems } \\
\hline Imipenem & $7(35)$ & $11(73.3)$ & $4(33.3)$ \\
\hline Meropenem & $4(20)$ & $5(33.3)$ & $3(25)$ \\
\hline Ertapenem & $5(25)$ & $4(26.6)$ & NA \\
\hline Doripenem & $5(25)$ & $2(13.3)$ & $1(8.3)$ \\
\hline \multicolumn{4}{|l|}{ Phenicols } \\
\hline Chloramphenicol & $4(20)$ & $2(13.3)$ & NA \\
\hline \multicolumn{4}{|l|}{ Folate pathway inhibitors } \\
\hline Co-trimoxazole & $12(60)$ & $10(66.6)$ & $2(16.7)$ \\
\hline \multicolumn{4}{|l|}{ Tetracyclines } \\
\hline Doxycycline & $3(15)$ & $2(13.3)$ & $2(16.7)$ \\
\hline
\end{tabular}

Abbreviations: NA, not applicable; $n$, number of resistant isolates; $N 1, N 2, N 3$, total number of isolates of Klebsiella spp., Escherichia coli and Acinetobacter spp., respectively

spectrum antibiotics given as treatment or prophylaxis exert selection pressure leading to the survival of resistant strains of bacteria, particularly in the gut. ${ }^{36}$

Some of the limitations of this study were as follows: (1) Speciation of microbial isolates could not be performed as conventional methods of microbial identification were used. (2) Anaerobic blood culture was not done owing to infrastructure-related issues. (3) Special growth conditions for fastidious organisms could not be provided owing to non-availability of special media. (4) Specific resistance categories like methicillin resistance, vancomycin resistance, extended-spectrum beta-lactamases, metallo$\beta$-lactamases, etc., were not looked into. (5) Antifungal susceptibility testing could not be performed. (6) Treatment outcomes of the patients were not analyzed.

It is evident from the results of this study that BSIs due to antibiotic-resistant bacteria are prevalent in FN patients receiving treatment at our center. BSIs with MDR pathogens in FN patients can lead dire complications and high mortality rates. Implementation of antimicrobial stewardship program along with evidence-based hospital infection control practices is the need of the hour. 
Table 5 Percentage antibiotic resistance pattern of coagulase negative Staphylococci $(N=20)$

\begin{tabular}{|l|l|}
\hline Antibiotics & $\begin{array}{l}\text { Coagulase negative } \\
\text { Staphylococcus spp. } \boldsymbol{n} \text { (\%) }\end{array}$ \\
\hline Aminoglycosides & NA \\
\hline Amikacin & NA \\
\hline Tobramycin & $8(40)$ \\
\hline Gentamicin & $7(35)$ \\
\hline Macrolides & $10(50)$ \\
\hline Azithromycin & $12(60)$ \\
\hline Erythromycin & $13(65)$ \\
\hline Fluoroquinolones & $18(90)$ \\
\hline Ciprofloxacin & $8(40)$ \\
\hline Ofloxacin & $11(55)$ \\
\hline Penicillins & $9(45)$ \\
\hline Ampicillin & $6(30)$ \\
\hline Lincosamides & \\
\hline Clindamycin & \\
\hline Oxazolidinones & \\
\hline Linezolid & \\
\hline Tetracyclines & \\
\hline Doxycycline & \\
\hline Phenicols & \\
\hline Chloramphenicol & \\
\hline Folate pathway inhibitors & \\
\hline Co-trimoxazole & \\
\hline
\end{tabular}

Abbreviations: NA, not applicable; $n$, number of resistant isolates; $N$, total number of isolates.

\section{Source(s) of Support}

None.

\section{Conflict of Interest}

None.

\section{References}

1 Freifeld AG, Bow EJ, Sepkowitz KA, et al; Infectious Diseases Society of America. Clinical practice guideline for the use of antimicrobial agents in neutropenic patients with cancer: 2010 update by the Infectious Diseases Society of America. Clin Infect Dis 2011;52(4):e56-e93

2 Klastersky J. Management of fever in neutropenic patients with different risks of complications. Clin Infect Dis 2004;39(suppl 1):S32-S37

3 Jacob LA, Lakshmaiah KC, Govindbabu K, et al. Clinical and microbiological profile of febrile neutropenia in solid tumors and hematological malignancies at a tertiary cancer care center in South India. Indian J Cancer 2014;51(4):464-468

4 Liu CY, Lai YC, Huang LJ, et al. Impact of bloodstream infections on outcome and the influence of prophylactic oral antibiotic regimens in allogeneic hematopoietic SCT recipients. Bone Marrow Transplant 2011;46(9):1231-1239

5 Penack O, Becker C, Buchheidt D, et al. Management of sepsis in neutropenic patients: 2014 updated guidelines from the Infectious Diseases Working Party of the German Society of Hematology and Medical Oncology (AGIHO) Ann Hematol 2014;93(7):1083-1095
6 Jeddi R, Achour M, Amor RB, et al. Factors associated with severe sepsis: prospective study of 94 neutropenic febrile episodes. Hematology 2010;15(1):28-32

7 Kang CI, Song JH, Chung DR, et al; Korean Network for Study of Infectious Diseases (KONSID). Risk factors and pathogenic significance of severe sepsis and septic shock in 2286 patients with gram-negative bacteremia. J Infect 2011;62(1):26-33

8 Trecarichi EM, Tumbarello M. Antimicrobial-resistant gram-negative bacteria in febrile neutropenic patients with cancer: current epidemiology and clinical impact. Curr Opin Infect Dis 2014;27(2):200-210

9 Paul M, Dickstein Y, Borok S, Vidal L, Leibovici L. Empirical antibiotics targeting Gram-positive bacteria for the treatment of febrile neutropenic patients with cancer. Cochrane Database Syst Rev 2014;1(1):CD003914

10 Amit S, Mishali H, Kotlovsky T, Schwaber MJ, Carmeli Y. Bloodstream infections among carriers of carbapenem-resistant Klebsiella pneumoniae: etiology, incidence and predictors. Clin Microbiol Infect 2015;21(1):30-34

11 Ortega M, Marco F, Soriano A, et al. Analysis of 4758 Escherichia coli bacteraemia episodes: predictive factors for isolation of an antibiotic-resistant strain and their impact on the outcome. J Antimicrob Chemother 2009;63(3):568-574

12 Viale P, Giannella M, Lewis R, Trecarichi EM, Petrosillo N, Tumbarello M. Predictors of mortality in multidrug-resistant Klebsiella pneumoniae bloodstream infections. Expert Rev Anti Infect Ther 2013;11(10):1053-1063

13 Feld R. Bloodstream infections in cancer patients with febrile neutropenia.IntJAntimicrob Agents 2008;32(suppl1):S30-S33

14 Perez F, Adachi J, Bonomo RA. Antibiotic-resistant gram-negative bacterial infections in patients with cancer. Clin Infect Dis 2014;59(suppl 5) :S335-S339

15 Collee JG, Miles RS, Watt B, Tests for the identification of bacteria. In: Collee JG, Fraser AG, Marimon BP, Simmons A, eds. Mackie \& Mc Cartney Practical Medical Microbiology. 14th ed. New Delhi: Elsevier; 2007 (reprint):131-149

16 Clinical and Laboratory Standards Institute, Performance Standards for Antimicrobial Susceptibility Testing; 29th Informational Supplement. CLSI Document M100. Wayne, PA: Clinical and Laboratory Standards Institute; 2019

17 European Committee on Antimicrobial Susceptibility Testing (EUCAST). Breakpoint tables for interpretation of MICs and zone diameters. Version 2019:9. Available at: http://www. eucast.org. Accessed December 30, 2019

18 Sickles EA, Greene WH, Wiernik PH. Clinical presentation of infection in granulocytopenic patients. Arch Intern Med 1975;135(5):715-719

19 Safdar A, Armstrong D. Infections in patients with hematologic neoplasms and hematopoietic stem cell transplantation: neutropenia, humoral, and splenic defects. Clin Infect Dis 2011;53(8):798-806

20 Babu KG, Lokanatha D, Lakshmaiah KC, et al. Bloodstream infections in febrile neutropenic patients at a tertiary cancer institute in South India: a timeline of clinical and microbial trends through the years. Indian J Med Paediatr Oncol 2016;37(3):174-182

21 Parikh S, Goswami P, Anand A, et al. Clinical and microbiological profile of infections during induction phase of acute myeloid leukemia. Gulf J Oncolog 2018;1(27):18-23

22 Whittle SB, Williamson KC, Russell HV. Incidence and risk factors of bacterial and fungal infection during induction chemotherapy for high-risk neuroblastoma. Pediatr Hematol Oncol 2017;34(5):331-342

23 El-Mahallawy HA, Hassan SS, El-Wakil M, Moneer MM, Shalaby L. Increasing antimicrobial resistance monitored in surveillance analysis of blood stream infections in febrile neutropenic pediatric oncology patients. Asian Pac J Cancer Prev 2015;16(14):5691-5695 
24 El-Mahallawy HA, El-Wakil M, Moneer MM, Shalaby L. Antibiotic resistance is associated with longer bacteremic episodes and worse outcome in febrile neutropenic children with cancer. Pediatr Blood Cancer 2011;57(2):283-288

25 Esbenshade AJ, Pentima MC, Zhao Z, et al. Development and validation of a prediction model for diagnosing blood stream infections in febrile, non-neutropenic children with cancer. Pediatr Blood Cancer 2015;62(2):262-268

26 Horasan ES, Ersoz G, Tombak A, Tiftik N, Kaya A. Bloodstream infections and mortality-related factors in febrile neutropenic cancer patients. Med Sci Monit 2011;17(5):CR304-CR309

27 Lubwama M, Phipps W, Najjuka CF, et al. Bacteremia in febrile cancer patients in Uganda. BMC Res Notes 2019;12(1):464

28 Kamonrattana R, Sathitsamitphong L, Choeyprasert, W, Charoenkwan P, Natesirinilkul R, Fanhchaksai K. A randomized, open-labeled, prospective controlled study to assess the efficacy of frontline empirical intravenous piperacillin/ tazobactam monotherapy in comparison with ceftazidime plus amikacin for febrile neutropenia in pediatric oncology patients. APOCP 2019;20:2733-2737

29 Noronha V, Joshi A, Patil VM, et al. Pattern of infection, therapy, outcome and risk stratification of patients with febrile neutropenia in a tertiary care oncology hospital in India. Indian J Cancer 2014;51(4):470-474

30 Mandal PK, Maji SK, Dolai TK, et al. Micro-organisms associated with febrile neutropenia in patients with haematological malignancies in a tertiary care hospital in Eastern India. Indian J Hematol Blood Transfus 2015;31(1):46-50
31 Kokkayil P, Agarwal R, Mohapatra S, et al. Bacterial profile and antibiogram of blood stream infections in febrile neutropenic patients with haematological malignancies. J Infect Dev Ctries 2018;12(6):442-447

32 Rose W, Veeraraghavan B, George B. Bloodstream infections in children with febrile neutropenia: Isolates and their antimicrobial susceptibility profile. Indian J Cancer 2015;52(4):495-496

33 Singhal T, Shah S, Naik R. The microbial etiology and antimicrobial susceptibility of bloodstream infections in patients with cancer at a private tertiary care hospital in Mumbai, India. Indian J Cancer 2016;53(3):452-453

34 Karanwal AB, Parikh BJ, Goswami P, Panchal HP, Parekh BB, Patel KB. Review of clinical profile and bacterial spectrum and sensitivity patterns of pathogens in febrile neutropenic patients in hematological malignancies: a retrospective analysis from a single center. Indian J Med Paediatr Oncol 2013;34(2):85-88

35 Daef EA, Elsherbiny NM, Agban MN, Riad KF, Mohammed LF. bloodstream infections in febrile neutropenic pediatric cancer patients: microbiological and sepsis biomarkers insight. Egypt J Immunol 2018;25(2):21-34

36 Nouér SA, Nucci M, Anaissie E. Tackling antibiotic resistance in febrile neutropenia: current challenges with and recommendations for managing infections with resistant gram-negative organisms. Expert Rev Hematol 2015;8(5):647-658 